\title{
Was der Mensch braucht
}

\author{
Die Qualitätsziele der Freien Wohlfahrtspflege im Lichte von \\ Gesellschaft und Organisation
}

Christoph Langer

\begin{abstract}
Anliegen des Qualitätsmanagements ist verbindlich festzulegen und öffentlich transparent zu machen, wie eine Organisation oder ein Unternehmen arbeitet. Dieser Aufgabe muss sich auch professionelle Soziale Arbeit stellen.
\end{abstract}

In dieser Zeitschrift hat sich Prof. Dr. Silvia Staub-Bernasconi kritisch mit den Qualitätszielen der Freien Wohlfahrtspflege auseinandergesetzt (vgl. Blätter der Wohlfahrtspflege 6/2010). Sie richtete ihr Hauptaugenmerk dabei insbesondere auf die Frage, ob die von der Freien Wohlfahrtspflege formulierten Qualitätsziele mit den von ihr vertretenen Prinzipien für professionelles Handeln vereinbar sind. An diesen Diskurs will dieser Beitrag anknüpfen.

Der von Staub-Bernasconi propagierte Ansatz, dem das sogenannte prozessualsystematische Paradigma zugrunde liegt, geht davon aus, dass alles Existente

- in Systeme eingewoben und

- in Bewegung, vergänglich und veränderbar, also Prozessen unterworfen ist. Diese Sichtweise weist Parallelen mit den Ansätzen des Qualitätsmanagements auf, das ebenfalls prozessual-systematisch angelegt ist. Nicht nur, weil es die Abläufe und Funktionsweisen der Organisation beschreibt und regelmäßig durch Audits auf ihre Deckung mit der Praxis überprüft und gegebenenfalls korrigiert. Sondern auch, weil die Anwendung des PDCA-Zyklus zu einem kontinuierlichen Verbesserungsprozess führt: Die Organisation ist ständig im Fluss und passt die Erfordernisse den Bedürfnissen an. Die

Christoph Langer leitet die Arbeitsstelle Qualitätsmanagement/ Qualitätspolitik im Deutschen Caritas-verband e. V. Der Beitrag entstand in Abstimmung mit dem Ausschuss Qualitätsmanagement der Bundesarbeitsgemeinschaft der Freien Wohlfahrtspflege.

E-Mail Christoph.Langer@caritas.de
Prinzipien des professionellen Handelns nach Staub-Bernasconi und des Qualitätsmanagements lassen sich gegenüberstellen (vgl. Tabelle S. 189).

Beim Qualitätsmanagement ließe sich einwenden, dass es den Ablauf eines Teilprozesses beschreibt, zum Beispiel die Beratung von Kundinnen und Kunden oder die Einstellung neuer Mitarbeitender, nicht aber die einzelne Aktion als solche. Dieser Einwand mag auf den ersten Blick richtig erscheinen. Er lässt jedoch außer Acht, dass an dieser Schnittstelle Qualitätsmanagement und Fachlichkeit bzw. professionelles Handeln ineinandergreifen: Qualitätsmanagement strukturiert das Handeln in der Organisation und ihrer Mitglieder, professionelles Handeln nach Staub-Bernasconi hingegen strukturiert das professionelle Vorgehen im einzelnen Beratungsfall. Somit ergänzt das eine das andere.

Beim Qualitätsmanagement sind die von Staub-Bernasconi propagierten Kriterien des professionellen Handelns folgendermaßen berücksichtigt:

- Wissenschaftsbasierung der Handlungsvollzüge: Klare und verständliche Regelungen der Handlungsabläufe werden - wenn möglich bzw. bereits erforscht - mit wissenschaftlich evaluierten Kenngrößen hinterlegt und messbar gemacht (die Messbarkeit und wissenschaftliche Basierung von Ergebnisqualität steht zugegebenermaßen noch am Anfang).

- Menschenrecht und soziale Gerechtigkeit: Menschenrechte sind eine unumstößliche Grundlage allen Handelns unserer Gesellschaft. Die Herbeiführung von sozialer Gerechtigkeit ist ein essentielles Anliegen der Freien Wohlfahrtspflege und der Leitbilder ihrer Einrichtungen. Das Qualitätsmanagementsystem trägt diese ethischen Leitlinien zusätzlich in die Organisation und verankert sie dort.

- Weisungsgebundenheit an den Träger: Ist gegeben, aber im Rahmen von klaren Regelungen und Verantwortlich- keiten, selten oder kaum hingegen in Bezug auf fachliche Belange in der (Einzelfall-)Beratung.

Schon dieser Vergleich verdeutlicht, dass Qualitätsmanagement die von Staub-Bernasconi propagierten Kriterien für Profession berücksichtigt und grundsätzlich erfüllt. Ausgehend von diesem Befund wird im Folgenden die von ihr angeführte Kritik zu den wichtigsten Grundanliegen professionellen Handelns beim Qualitätsmanagement näher beleuchtet.

\section{Leitbildorientierung}

In Bezug auf die Leitbildorientierung argumentiert Staub-Bernasconi, es sei aufgrund des Textes nicht erkennbar, ob an diesem Leitbild auch Sozialarbeiterinnen und Sozialarbeiter mitgewirkt haben bzw. wer ein Anrecht auf Mitbestimmung bei dessen Weiterentwicklung hat.

Die Freie Wohlfahrtspflege hat den Selbstanspruch, möglichst alle Akteure auf Führungs- und Mitarbeitendenebene an der Leitbildentwicklung zu beteiligen: Vorstand, Leitungskräfte, Sozialarbeiterinnen und Sozialarbeiter, Beratende, Verwaltungsmitarbeiterinnen und Verwaltungsmitarbeiter, Servicekräfte für Reinigung, Verpflegung etc. - um nur einige zu nennen. Es liegt ihr keinesfalls daran, eine Berufsgruppe in irgendeiner Weise herauszustellen. Demnach werden Sozialarbeiterinnen und Sozialarbeiter von der Leitbildentwicklung nicht ausgeschlossen, sondern an dieser beteiligt. Nicht zuletzt, weil diese Berufsgruppe zu den zentralen Leistungsträgern der Sozialen Arbeit gehört.

\section{Orientierung am persönlichen Nutzen}

Staub-Bernasconi kritisiert, die Freie Wohlfahrtspflege gehe offensichtlich da- 
von aus, der Mensch, der eine Einrichtung aufsucht, werde in erster Linie nicht mehr als Mensch gesehen, sondern als Kunde, der eine Leistung nutzen möchte. Dass dieser Mensch als erstes ein Hilfe benötigender Mensch in Not sein könnte, sei nicht angedacht.

Genau dies aber ist angedacht! Viele Einrichtungen sind bestrebt, möglichst einfache Zugänge zu den von ihnen angebotenen Diensten zu schaffen. Jedoch betrachten sie dabei einen Menschen, der aus welchen Gründen auch immer eine Beratungsstelle um Hilfe aufsucht, nicht als ein gänzlich handlungsunfähiges Subjekt. Als solches hätte er die Beratungsoder Hilfestelle gar nicht aufsuchen können! Er mag in manchen Bereichen Schwächen und Defizite haben, ist aber trotzdem ein entscheidungs- und handlungsfähiges Individuum.

Die Freie Wohlfahrtspflege spricht in ihrem Grundsatzpapier »Die Qualitätsziele der Freien Wohlfahrtspflege " von »Kund(inn)en «. Nicht, weil ein neoliberales Wirtschafts- und Menschenbild mittlerweile zur Handlungsgrundlage geworden wäre. Es geht vielmehr darum, den vielfältigen Entwicklungen Rechnung zu tragen. Sei es, indem soziale Leistungen, beispielsweise Pflege - wie vom Gesetzgeber ausdrücklich gewünscht -, auf einem am Wettbewerb orientierten Markt angeboten werden. Vor allem aber soll dieser Begriff unser Menschenbild deutlich machen: Wir sehen jeden, der zu uns kommt, als Person und Subjekt, und nicht als Objekt, das unsere Hilfeleistungen empfangen darf.

Ein hilfebedürftiger Mensch, der eine Beratung sucht, hat für sich erkannt, dass er Hilfe braucht. Er will einem von ihm empfundenen Mangel etwas entgegensetzen, um seine Lebensqualität zu verbessern. Um aber eine maßgeschneiderte Beratung oder Therapie vornehmen zu können, ist es unerlässlich, eine Anamnese durchzuführen: Wie ist die aktuelle Lebenssituation des Hilfebedürftigen? Wo liegt das Problem bzw. sind die Probleme begründet? Was hat ihn motiviert, Hilfe zu suchen? Was will er damit erreichen?

Fragen wie diese geben die Messlatte für die Beratung vor. Dabei fallen nicht nur die Ziele und Vorstellungen des Ratsuchenden ins Gewicht, sondern auch die Expertise der beratenden Fachkräfte, das heißt, was sie aufgrund ihrer Kompetenz für geboten erachten. Ihre Zielvorstellun- gen können zugegebenermaßen bisweilen von denen der Kunden abweichen. In solchen Fällen klären beide Seiten ihre Standpunkte $\mathrm{ab}$ und verständigen sich auf ein gemeinsames Ziel. Dieser Verständigungsprozess mündet in einen Therapie- oder Maßnahmenplan, aus dem hervorgeht, wie dieses Ziel erreicht werden soll. Solches Vorgehen stellt einen wichtigen Aspekt von Qualität im gesamten Beratungsprozess dar - neben vielen anderen.

Als weiteres Argument zu diesem Grundanliegen fragt Staub-Bernasconi, wie es komme, dass Wohlfahrtsverbände sich ausgerechnet dem Bild eines Menschen verschreiben und unterordnen, der - ohne sozialen Bezug auf Mitmenschen offenbar nichts anderes im Sinn hat, als auf einem Dienstleistungsmarkt seinen individuellen Nutzen in Form von Wünschen zu verfolgen. So werde hier »Qualität « mit individueller Nutzenmaximierung gleichgesetzt.

Diese Kritik von Staub-Bernasconi richtet sich offenbar an den Qualitätsmanagementansatz der Bundesarbeitsgemeinschaft der Freien Wohlfahrtpflege. Ausgehend von ihrem Ansatz richtet sie hier wohl ihr Augenmerk auf die Ziele der Sozialen Arbeit. Diese sind:

- individuelle Bedürfnis- und Wunscherfüllung

- fairer Ausgleich von Rechten und Pflichten zwischen Menschen und sozialen Gruppen und Regeln der Machtbegrenzung.

In diesem Punkt aber sind die Grundgedanken von Staub-Bernasconi und der Freien Wohlfahrtspflege deckungsgleich und haben die gleiche Zielrichtung. Hinzuzufügen wäre allerdings, dass zu den Adressaten der Arbeit der Freien Wohlfahrtspflege auch Menschen mit ihren Sorgen und Nöten aus dem Mittelstand gehören. Auch sie haben Probleme bei der Erziehung von Kindern, einen Schwangerschaftskonflikt oder ein Suchtproblem. Was die Wissenschaftlerin in ihrem Diskurs auch bestätigt, wenn sie argumentiert, Soziale Arbeit sei gemäß ihrem nationalen und internationalen Ethikkodex verpflichtet, die Bedürfnisbefriedigung ihrer Adressatinnen und Adressaten und damit ihr Wohlbefinden zu ermöglichen, wenn sie dies nicht aufgrund eigener, individueller Ressourcen und des diskriminierungsfreien Zugangs zum Bildungs-, Wirtschafts- und kulturel- len System tun können, weil sie sozialstrukturellen Unrechtserfahrungen unterworfen sind.

\section{Orientierung an Gemeinwesen und Gesellschaft}

Bei diesem Grundanliegen professionellen Handelns kritisiert die Autorin, werde die Marschrichtung beim Qualitätsmanagement vornehmlich aus der Perspektive der Organisation vorgegeben. Demnach werde die Verankerung der Organisation im Gemeinwesen als Führungsaufgabe verstanden, nicht jedoch als klassische Aufgabe von Sozialarbeitenden, in demokratisch-partizipativer Zusammenarbeit.

Nach dem Verständnis der Freien Wohlfahrtspflege ist die Verankerung der Organisation tatsächlich eine Führungsaufgabe, insbesondere aus folgenden Gründen:

- Die Marschrichtung muss dem erklärten und gewollten Ziel der Einrichtung entsprechen, um den Mitarbeiterinnen und Mitarbeitern einen Orientierungsrahmen für ihre Tätigkeit zu geben.

- Dies ermöglicht, ihre Aktivitäten einem vorgegebenen Konzept folgend zielgerichtet aufeinander abzustimmen.

- Auf diese Weise erhalten die Mitarbeiterinnen und Mitarbeiter klare Zielvorgaben für die Umsetzung ihrer Arbeit. In diesem Sinne bezieht sich Führungsaufgabe nicht auf die konkrete Umsetzung der Vernetzung im Gemeinwesen, sondern vielmehr auf die Überwachung des Erreichens der anvisierten Ziele.

Staub-Bernasconi argumentiert des Weiteren, in den Qualitätszielen der Freien Wohlfahrtspflege sei nirgendwo festgehalten, dass sich Stadtteilbewohner zusammentun, um - befähigt und unterstützt durch professionelle Sozialarbeiterinnen und Sozialarbeiter - die Beschaffung von Informationen, die Formulierung von Bedürfnissen, Interessen, Zielen und die Verteilung von Aufgaben zur Problemlösung zu übernehmen.

Dem Verständnis der Freien Wohlfahrtspflege zufolge kann eine Organisation oder eine Fachkraft nur dann demokratisch-partizipative Zusammenarbeit im Stadtteil realisieren und unterstützen, wenn die Bewohnerinnen und Bewohner dies wünschen und wenn konkreter Bedarf nach dieser besteht. Dieser Sachver- 
halt ist in den Qualitätszielen nicht so explizit formuliert, wie von Staub-Bernasconi gefordert, dafür aber in den Qualitätsanforderungen zur Gemeinwesenund Bürgerorientierung berücksichtigt:

- „Die Einrichtung/Organisation versteht sich als Bestandteil und Mitgestalter des Sozialraums.

- Die Einrichtung/Organisation arbeitet aktiv an der Vernetzung im Gemeinwesen mit.

- Die Einrichtung unterstützt Bürgerinnen und Bürger in ihrem Vorhaben, sich freiwillig zu engagieren." (z. B. Leitlinien zur Qualität, zum Umwelt- und Arbeitsschutz) als auch die konkrete Festlegung der Ziele und der Maßnahmen zu deren Erreichung.

Do: Die Managementnormen enthalten neben Vorgaben für die konsequente Umsetzung der festgelegten Maßnahmen auch Regelungen zum Managementsystem, zum Beispiel für den Aufbau der Einrichtung (Struktur, Aufgaben und Verantwortlichkeiten, ggf. Schulungsmaßnahmen für die zuständigen Mitarbeiterinnen und Mitarbeiter) und den Ablauf ihrer relevanten Aufgaben. Doku-

\section{"Qualitätsmanagement geht es letztlich um den Ausgleich verschiedener Interessen "}

Diese Qualitätsanforderungen gehen ausdrücklich auf die von Staub-Bernasconi geforderte Unterstützung der Bürgerinnen und Bürger ein. Ihre Formulierung jedoch ist bewusst offen gehalten, weil die Qualitätsziele der Bundesarbeitsgemeinschaft der Freien Wohl-fahrtsverbände für alle Arten von Einrichtungen richtungsweisend sind.

\section{Ziel- und Wirkungsorientierung}

Staub-Bernasconi wendet ein, der als Prüfkriterium definierte »Regelkreislauf « verweise am Klarsten auf die Weichenstellung zwischen dem eingangs beschriebenen Berufs- versus Professionsszenario. Da er mit der "Zielformulierung « beginnt, müsse vermutet werden, dass auf eine Beschreibung und Erklärung der Problemsituation - beides ihrer Meinung nach unhintergehbare Anforderungen an eine Profession - verzichtet werden kann, weil es um die Zuordnung zwischen zumeist standardisierten (u. a. rechtlichen) »Maßnahmen « und »Wünschen « geht.

Dieser von Staub-Bernasconi angesprochene Aspekt hebt sehr stark auf den PDCA-Zyklus und dessen konsequente Umsetzung ab. Dieser Zyklus lässt sich wie folgt beschreiben:

Plan: Planung beinhaltet zwingend die Festschreibung der Grundsätze der Einrichtung in dokumentierten Leitlinien mentationen wie diese dienen zur Sicherstellung der Verbindlichkeit und Nachvollziehbarkeit (vgl. Zollondz 2001: 488).

Check: Bereits bei der Umsetzung wird regelmäßig überprüft, ob die bislang durchgeführten Maßnahmen zielkonform sind.

Act: Ist dies nicht der Fall, wird nach den Ursachen für die Schwachstellen geforscht und nach Lösungen gesucht, um die anvisierten Ziele doch noch zu erreichen.

Der PDCA-Zyklus kommt bei allen relevanten Aktivitäten zum Tragen, insbesondere eben auch bei der Anwendung des Qualitätsmanagementsystems, aber letztendlich auch im Beratungsprozess.

Leider arbeiten soziale Einrichtungen in der Praxis nicht immer (konsequent) nach dem PDCA-Zyklus. Manche verzichten sogar ganz auf konkrete Zielformulierungen: Sie "wursteln sich durch «, je nach Maßgabe, was aktuell nachgefragt oder finanziert wird. Es geht letztlich darum, diese Einrichtungen anzuregen, Zielvorgaben zu formulieren und sich nach diesen auszurichten.

Voraussetzung für professionelle Zielformulierung jedoch ist die Durchführung einer Ist-Analyse. In diesem Punkt sind die Qualitätsanforderungen der Bundesarbeitsgemeinschaft der Freien Wohlfahrtspflege tatsächlich nicht präzise genug formuliert und bedürfen bei an- stehender Revision einer Ergänzung. Eines allerdings bleibt festzuhalten: Das Papier der Bundesarbeitsgemeinschaft der Freien Wohlfahrtspflege zielt keinesfalls auf eine Entprofessionalisierung durch Qualitätsmanagement ab, wie Staub-Bernasconi wegen der fehlenden Auflistung der Ist-Analyse unterstellt. Im Gegenteil: Es ist ausdrücklich gewollt, die Profilierung der Dienste und Einrichtungen zu schärfen und die Mitarbeitenden in ihrer Professionalität zu stärken. Die Anwendung des PDCA-Zyklus unterstreicht den Anspruch der Profession und der Professionalisierung.

\section{Vertragspartnerschaft}

Staub-Bernasconi kritisiert, einseitige Informationsabgabe könne wohl kaum als »Partnerschaft " im Rahmen einer »koproduktiven Arbeitsbeziehung « bezeichnet werden. Aus professioneller Perspektive müssten die abzuschließenden Verträge verfassungs- und menschenrechtskonform sein. Für die Autorin ist dies das wichtigste Qualitätskriterium. Folgerichtig könne die Kürzung von Sozialhilfe unter das Existenzminimum oder gar auf Null aus philosophisch-menschenrechtlicher, aber auch aus bedürfnistheoretischer Sicht in keiner Weise legitimiert werden. Aus welchem Grund auch immer sie vorgenommen werde.

Dem ist zu entgegnen, dass das Kriterium einer Vertragspartnerschaft nur auf einen Teil der Nutzerinnen und Nutzer zutrifft. Zugegebenermaßen wird hier in der Praxis nicht sorgfältig genug unterschieden zwischen Verträgen mit Nutzern und solchen mit Lieferanten oder Koproduzenten. Letztlich geht es um die Stärkung der Verbraucherrechte, das heißt der Rechte der Nutzerinnen und Nutzer. Es ist doch unstrittig, dass Verträge - wie von Staub-Bernasconi als Kriterium für Professionalität gefordert - verfassungsund menschenrechtskonform sein müssen. Dies ist Grundlage für jeden Vertragsabschluss und Rechtsverkehr in unserem Rechtsstaat.

Diese Verfassungs- und Menschenrechtskonformität trifft auf Individualverträge zu. Davon natürlich strikt zu trennen sind Aktivitäten und Maßnahmen des Gesetzgebers. Die Kürzung der Sozialhilfe durch den Gesetzgeber, die Staub-Bernasconi hier anspricht, unter- 
liegt anderen Mechanismen, weil sie eine gesetzliche Vorgabe darstellt, auf die eine Einrichtung keinen Einfluss ausüben kann - schon gar nicht durch das Qualitätsmanagementsystem. Insofern hat solch eine gesetzliche Vorgabe auch keinen Einfluss auf einen (möglichen) Vertragsabschluss zwischen Leistungserbringer und Nutzern. Dass die Wohlfahrtsverbände bei möglichen Kürzungen sozialstaatlicher Leistungen als Anwalt der Betroffenen auftreten, ist selbst erklärtes Ziel. Ob dies immer gelingt, sei dahin gestellt. Dieser Aspekt jedoch steht in keinem Zusammenhang mit der Vertragspartnerschaft.

\section{Ressourcenorientierung}

Der Hinweis auf die europäisch anerkannten Normen (DIN EN ISO 9001:2000; EFQM-Modell für Excellence) für das Qualitätsmanagement führt Staub-Bernasconi zu der Annahme, es bestehe eine Gewichtung zugunsten der Betriebswirtschaft.
Staub-Bernasconi wähnt eine zunehmende Dominanz der Betriebswirtschaft bei den Wohlfahrtsverbänden, erachtet diese gar als das einzig bestimmende Kriterium. Das mag zu einem gewissen Teil auch zutreffen, dann aber aus triftigem Grund: Zuschüsse werden laufend reduziert oder fallen aufgrund der angespannten Finanzlage vieler Kostenträger gänzlich weg. Unstrittig aber ist: Jede Organisation kann nur bestehen, wenn sie wirtschaftlich arbeitet und nicht mehr Mittel ausgibt, als ihr zur Verfügung stehen, denn auch Wohlfahrtsverbände sind Unternehmungen, die mit ihren Mitteln und Ressourcen haushalten müssen.

\section{Qualitätsmanagement basiert auf acht Säulen}

Dass es bei den Qualitätszielen der Freien Wohlfahrtspflege keineswegs schwerpunktmäßig um betriebswirtschaftliche oder organisationale Belange sowie deren Durchsetzung geht, wird bei genauerer Betrachtung der Grundlagen des Qualitätsmanagements deutlich. Hier fallen die » acht Säulen « des Qualitätsmanagements ins Auge. Diese sind in der 9000er Normenfamilie der DIN EN ISO beschrieben, finden sich aber auch - mit leicht modifizierter Terminologie - im Modell der European Foundation for Quality Management (EFQM) für Excellenz wieder. Es handelt sich hierbei um:

1. Kund(inn)enorientierung

2. Verantwortung der Führung

3. Einbeziehung der Personen/Mitarbeiter(innen)orientierung

4. Prozessorientierter Ansatz

5. Systemorientierter Managementansatz

6. Ständige Verbesserung

7. Sachbezogener Ansatz zur Entscheidungsfindung

8. Lieferantenbeziehungen zum gegenseitigen Nutzen

Der Betriebswirtschaft kommt hier keine herausragende Rolle zu. Zudem ergibt sich bei konsequenter Berücksichtigung und Umsetzung dieser »Säulen « eine Interaktion und Wechselwirkung mit den beteiligten Akteuren, die über rein organisationale oder betriebswirtschaftliche

\begin{tabular}{|l|l|}
\hline Professionelles Handeln nach Staub-Bernasconi & Qualitätsmanagement \\
\hline Prinzip: & Prinzip: \\
Zielrealisierung und problembezogenes Arbeiten & $\begin{array}{l}\text { Zielsetzung für die Organisation, für die in ihr stattfinden- } \\
\text { den verschiedenen Teilprozesse und für die Arbeit mit den }\end{array}$ \\
& Kundinnen und Kunden \\
\hline Handlungsmodell mit folgenden Schritten: & Handlungsmodell mit folgenden Schritten: \\
1. Was? & 1. Plan \\
Beschreibung des sozialen Problems mittels Daten & - Identifizierung des Problems \\
2. Warum? & - Definition/Festlegung eines Ziels/von Zielen \\
Erklärung des sozialen Problems durch wissenschaftliche & - Entwicklung eines Handlungsplans: Wer macht was wie \\
Theorien & womit und wann? \\
3. Woraufhin? & 2. Do \\
Bewertung des sozialen Problems und Zielsetzung für die & Ausführung des Planes: Wer macht was wie womit und \\
Lösung & wann? \\
4. Wer? & 3. Check \\
Bestimmung der Subjekte, die in die Problemlösung mitein- & Auswertung und Erfolgskontrolle: Bei Erreichen des \\
bezogen werden sollen; Formulierung von Aufgaben & Ziels/der Ziele hat sich das Vorgehen und die Methode be- \\
5. Womit? & währt \\
Bestimmung von Arbeitsweisen durch Methoden & 4. Act \\
6. Wo? & Bei Nichterreichen des Ziel/der Ziele: Zielkorrektur bzw. \\
Bestimmung von Handlungsweisen durch Pläne und Tech- & Anpassung der Maßnahmen \\
niken & \\
7. Ob? & \\
Auswertung und Erfolgskontrolle & \\
\hline
\end{tabular}

Die Prinzipien professionellen Handelns nach dem prozessual-systematischen Paradigma und des Qualitätsmanagements weisen durchaus Ähnlichkeiten auf. 
Interessen hinausgehen. Es sei denn, dass eine Interessengruppe die Oberhandhand gewinnt, wie es vielerorts in der Wirtschaft zu sehen ist, wo nur Shareholder Value zählt.

\section{Qualitätsziele professioneller Sozialer Arbeit}

Die Orientierung an den "acht Säulen « verhindert eine isolierte Perspektive von Sozialer Arbeit, sowohl aus Sicht der Organisation als auch der Adressaten Sozialer Arbeit, der Mitarbeitenden und der Betriebswirtschaft. Damit wäre zugleich eine Antwort auf den Einwand von Staub-Bernasconi gegeben, aufgrund des Qualitätsmanagements würden rein or-

\section{Literatur}

Deutscher Berufsverband für Soziale Arbeit (DBSH) e. V.: Grundlagen für die Arbeit des DBSH e. V. Schierling 2009.

Staub-Bernasconi, Silvia: Das fachliche Selbstverständnis Sozialer Arbeit. Wege aus der Bescheidenheit: Soziale Arbeit als Human Rights Profession. Soziale Arbeit im Wandel ihres Selbstverständnisses. Beruf und Identität. Hg. von Wolf Rainer Wendt. Freiburg im Breisgau 1995.

Staub-Bernasconi, Silvia: Soziale Arbeit: Dienstleistung oder Menschenrechtsprofession? Zum Selbstverständnis Sozialer Arbeit in Deutschland mit einem Seitenblick auf die internationale Diskussionslandschaft. Einführung in die Ethik der Sozialen Arbeit. Hg. von Walter Lesch und Andreas Lob-Hüdepohl. Paderborn, München, Wien, Zürich 2007.

Staub-Bernasconi, Silvia: Vom Beruf zur Profession. Die »Qualitätsziele der Wohlfahrtsverbände « im Lichte der Profession Sozialer Arbeit. Blätter der Wohlfahrtspflege 6/2010, 233-236.

Vorstand der Bundesarbeitsgemeinschaft der Freien Wohlfahrtspflege: Die Qualitätsziele der Freien Wohlfahrtspflege. Berlin 2005.

Zollondz, Hans-Dieter (Hg.): Lexikon Qualitätsmanagement. München 2001. ganisationale Interessen in Einrichtungen Oberhand gewinnen könnten.

Mit der Formulierung von Qualitätszielen hat die Freie Wohlfahrtspflege einen ersten kleinen Schritt zur Formulierung von Qualitätszielen professioneller Sozialer Arbeit unternommen. Darüber hinaus hat der Deutsche Berufsverband für Soziale Arbeit ein Grundraster zur Beurteilung der Qualität in den Handlungsfeldern Sozialer Arbeit herausgebracht. Denn gerade bei Sozialarbeiterinnen und Sozialarbeitern ist leider häufig eine $\mathrm{Ab}$ wehrhaltung gegen die Entwicklung und Umsetzung von Qualitätsmanagementzielen und Qualitätsmanagementsystemen zu beobachten. Mit dem Argument: »Wir arbeiten professionell, das reicht! «

Die schriftliche Fixierung dessen aber, wie gearbeitet wird - und letztendlich ist Qualitätsmanagement nicht anderes -, schafft Transparenz und macht die Professionalität Sozialer Arbeit sicht- und nachvollziehbarer. Daher ist in diesem Kontext das Anliegen von Staub-Bernasconi, Soziale Arbeit auf wissenschaftlich und professionsethisch fundierte Füße zu stellen, unbedingt zu unterstützen.

\section{Management und Kirche: Mit Gott rechnen.}

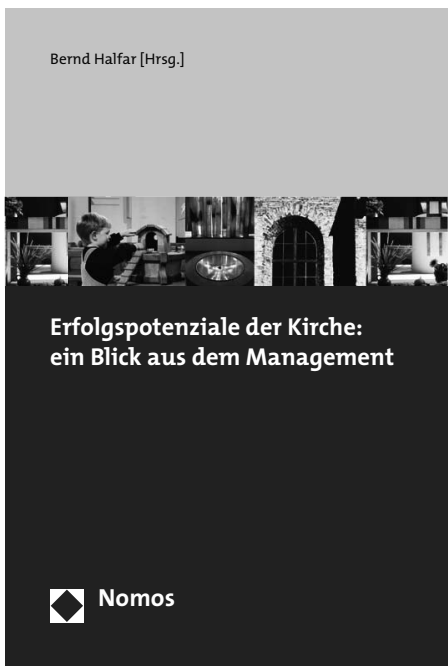

\section{Erfolgspotenziale der Kirche: ein Blick aus dem Management}

Herausgegeben von

Prof. Dr. Bernd Halfar

2011, ca. 220 S., brosch., ca. 29, $-€$

ISBN 978-3-8329-6451-1

Erscheint ca. September 2011

nomos-shop.de/13431

Das Buch zeigt praktische Verfahren, Instrumente, funktionierende Modelle und theoretische Besonderheiten aus dem Servicemanagement, der Religionssoziologie, dem strategischen Management, Finanzmanagement, Controlling, Personalmanagement und Kostenmanagement für die evangelische und katholische Kirche sowie die jüdischen Gemeinden auf.

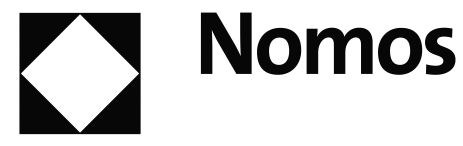

